'Physica Scripta. Vol. T49, 441-445, 1993

\title{
:Quantum Effects in Thermal and Thermo-Electric Transport in Semiconductor Nanostructures
}

\author{
L. W. Molenkamp, H. van Houten and A. A. M. Staring \\ Philips Research Laboratories, 5600 JA Eindhoven, The Netherlands \\ and \\ C. W. J. Beenakker \\ Instituut-Lorentz, University of Leiden, 2300 RA Leiden, The Netherlands
}

Received March 29. 1993; accepted April 21, 1993

\begin{abstract}
Current heating is used to create a micron-scale hot electron reservoir in semiconductor nanostructures at low temperatures. This technique enables the study of quantum effects in various thermo-electric transport coefficients.
\end{abstract}

\section{Introduction}

In the past few years, our understanding of transport phenomena in semiconductor nanostructures has increased considerably. For a general overview of transport phenomena in these devices, we refer the reader to Refs. [1] (quantum ballistic an quantum diffusive transport) and [2] (transport in the Coulomb blockade regime). In addition to the purely electronic transport phenomena discussed in these papers, also thermal and thermo-electric quantum-transport phenomena may be explored in semiconductor nanostructures. In the present article, we review our recent results on such effects in the quantum-ballistic transport regime, and in the Coulomb blockade regime.

Let us start by recapitulating the basics of thermoelectrics: An electrical current $I$ through a conductor is accompanied by a thermal current $Q$. These currents are driven by differences in voltage $(\Delta V)$ and temperature $(\Delta T)$ across the conductor. In the regime of linear response, this is conventionally expressed as [3]

$$
\begin{aligned}
-\Delta V & =R I+S \Delta T \\
Q & =\Pi I-\kappa \Delta T
\end{aligned}
$$

Here $R$ is the electrical resistance of the conductor, $S$ the thermopower, $\Pi$ the Peltier coefficient, and $\kappa$ the thermal conductance. The cross-coefficients $S$ and $\Pi$ are related by the Kelvin-Onsager relation

$\Pi=S T$.

For the experimentalist, the study of thermo-electrics in nanostructures represents a challenge: one needs to create an appreciable temperature difference (of the order of $1 \mathrm{~K}$ ) on a length scale of a few $\mu \mathrm{m}$. Clearly, it is impractical to use the heater-and-thermometer methods developed for bulk semiconductors. Instead, we have chosen to make use of a specific property of the semiconductors we study: in the two-dimensional electron gas (2DEG) in an (Al, Ga)As heterojunction structure at low temperatures (typically $\lesssim 2 \mathrm{~K}$ ), the coupling between hot electrons and the lattice is much smaller (typical relaxation time $\lesssim 1 \mathrm{~ns}$ ) than the coupling within the electron system ( $\sim$ ps). One can easily estimate that, when passing a current through a channel, a reservoir of hot electrons is created. It is then possible to study the thermoelectrics of nanostructures by suitably designing a sample structure around such a hot electron reservoir. Using this technique, we have been able to observe the quantum size effects in the thermopower [4], Peltier coefficient and thermal conductance [5] of a quantum point contact, as well as the sawtooth-shape oscillations of the thermopower of a quantum dot in the Coulomb blockade regime [6]. Examples of these experiments are given in this article.

\section{Experimental.}

The samples used for the experiments are defined electrostatically in the 2DEG of ( $\mathrm{Al}, \mathrm{Ga}) \mathrm{As}$ heterostructures. We use electron-beam lithography to fabricate TiAu gates of dimensions down to $0.1 \mu \mathrm{m}$. A typical (Al, Ga)As wafer has an electron density $n \approx 3 \times 10^{11} \mathrm{~cm}^{-2}$ and a mobility $\mu \approx 10^{6} \mathrm{~cm}^{2} /$ Vs. For transport measurements, the samples are kept in a cryostat at zero magnetic field. Measurements on quantum-ballistic transport are made at a temperature of $1.7 \mathrm{~K}$; experiments on the Coulomb blockade are performed in a dilution refrigerator at a temperature of $45 \mathrm{mK}$. The signals are measured using dc or low-frequency lock-in techniques.

\section{Thermopower of a quantum point contact}

First, we discuss the thermo-electric transport phenomena observed in the quantum-ballistic transport regime. This regime is most readily accessed using a quantum point contact (QPC), a short constriction of variable width, comparable to the Fermi wavelength. The most striking manifestation of quantum-ballistic transport is the quantization in units of $2 e^{2} / h$ of the electrical conductance of a QPC [7]. This quantization is observed as a series of plateaux in a plot of the conductance versus the voltage on the gate electrodes defining the constriction. The conductance quantum $2 e^{2} / h$ corresponds to a perfectly transmitted one dimensional (1D) subband in the QPC. Theoretically, it is clear that quantum size effects due to the $1 \mathrm{D}$ subband structure in a QPC should occur as well for the thermal conductance, the 
thermopower and the Peltier coefficient [3, 8-11]. Streda [9] has considered specifically the problem of the thermopower of a QPC. He found that the thermopower vanishes whenever the conductance of the point contact is quantized, and that it exhibits a peak between plateaux of quantized conductance.

An experimental arrangement for measuring the thermopower of a QPC is shown schematically in Fig. 1(a). By means of negatively biased split gates (A-F), a channel is defined in the 2DEG in an ( $\mathrm{Al}, \mathrm{Ga})$ As heterostructure. A QPC is incorporated in each channel boundary. The point contacts $\mathrm{AB}$ and $\mathrm{DE}$ face each other, so that the transverse voltage $V_{5}-V_{1}$ (measured using Ohmic contacts attached to the 2DEG regions behind the point contacts) does not contain a contribution from the voltage drop along the channel.

On passing a current $I$ between Ohmic contacts 6 and 3, the average kinetic energy of the electrons in the channel increases, because of the power dissipation. Due to frequent electron-electron scattering events, the electron distribution
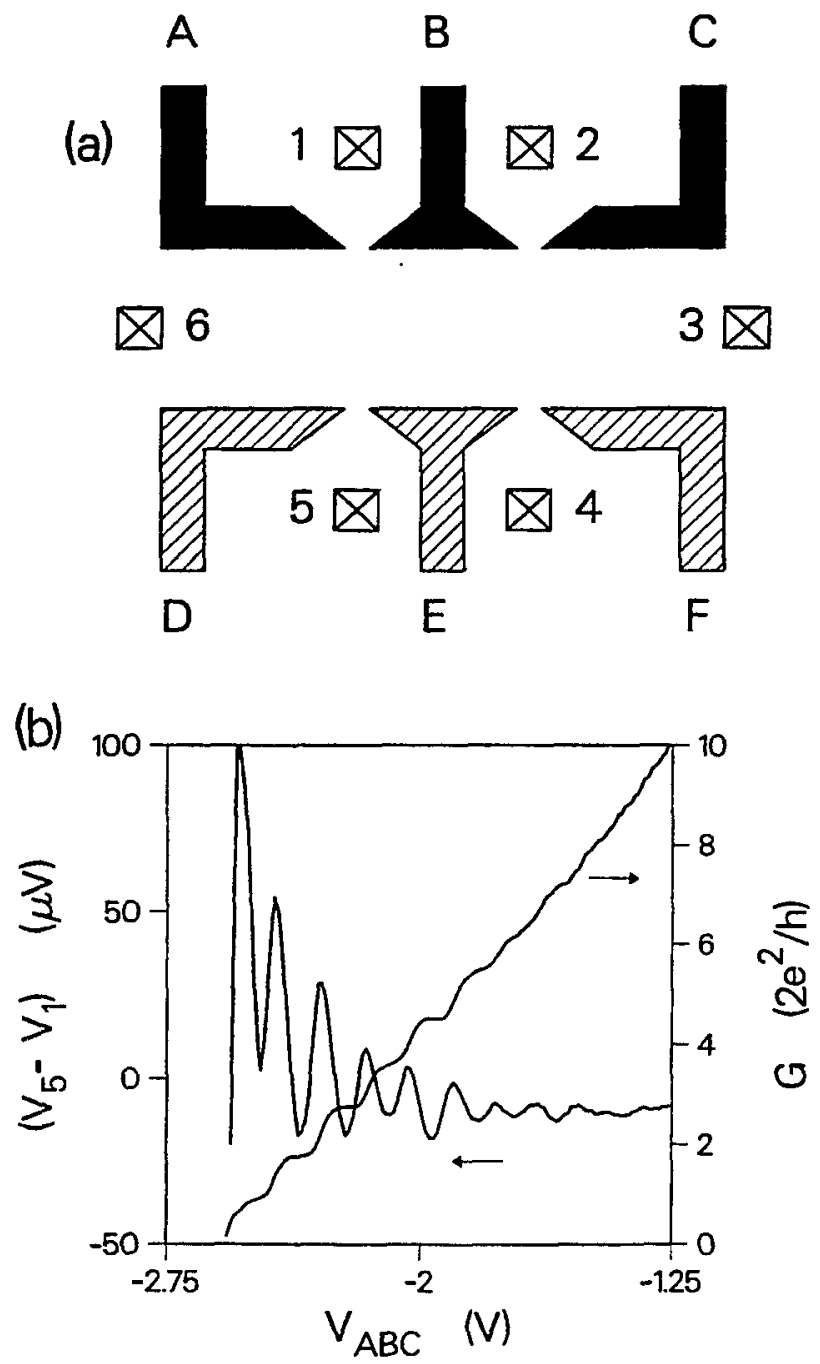

Fig. 1. (a) Schematic layout of the device used to demonstrate quantum oscillations in the thermopower of a QPC by means of a current heating technique. Hatched areas are gates, and crosses indicate Ohmic contacts. Black areas indicate gates whose voltage is varied in the experiment. The channel has a width of $4 \mu \mathrm{m}$ and a length of $20 \mu \mathrm{m}$. (b) Measured conductance and transverse voltage $\left(V_{5}-V_{1}\right)$ as a function of the gate voltage defining point contact 1 (black gates), at a lattice temperature of $1.65 \mathrm{~K}$ and a current of $5 \mu \mathrm{A}$. The gates defining point contact 2 (dashed) were kept fixed at $-2.0 \mathrm{~V}$. function in the channel thermalizes rapidly to a heated Fermi function at temperature $T+\Delta T$. Meanwhile, the regions of the $2 \mathrm{DEG}$ where no current is passed remain at electron temperature $T$. This means that we have created a temperature difference $\triangle T$ across all four QPC's $(A B, B C$, $\mathrm{DE}$, and $\mathrm{EF}$ ). Since the point contacts act as energy barriers for the electrons [1], this temperature difference induces thermovoltages across all QPC's: the cold 2DEG regions behind the QPC's charge up to counteract an influx of hot electrons from the channel. In our measuring configuration, this leads to the occurence of a transverse voltage $V_{\mathrm{S}}-V_{1}=\left(S_{\mathrm{DE}}-S_{\mathrm{AB}}\right) \Delta T$, where $S_{\mathrm{AB}}$ denotes the thermopower of QPC AB.

A typical experimental result [4] is shown in Fig. 1(b). In the experiments, the gate voltage on A, B and C [black in Fig. 1(a)] is varied, while the voltage on the other gates [hatched in Fig. 1(a)] is kept constant. In this way, any change in the transverse voltage $V_{5}-V_{1}$ is due to variations in $S_{\mathrm{AB}}$. [ $S_{\mathrm{DE}}$ is constant, but not negligible in this experiment, which is why the trace for $\left(V_{5}-V_{1}\right)$ drops below zero in Fig. 1(b).] Also shown is the conductance $G$ of QPC AB, obtained from a separate measurement. We observe strong oscillations in $V_{5}-V_{1}$. The peaks occur at gate voltages where $G$ changes stepwise because of a change in the number of occupied $1 \mathrm{D}$ subbands in QPC AB. These are the quantum oscillations of the thermopower predicted by Streda. [9] A detailed comparison of the oscillations in Fig. 1(b) with theory (extended to the regime of finite thermovoltages and temperature differences) has been presented elsewhere [4]. Here, we only point at the increase in peak height with decreasing subband index, a phenomenon predicted by Streda [9]. The largest peak near $G=1.5\left(2 e^{2} / h\right)$ has a measured amplitude of about $75 \mu \mathrm{V}$. The theoretical result of Ref. [9] predicts $S \approx-40 \mu \mathrm{V} / \mathrm{K}$ for this peak, which indicates that the temperature of the electron gas in the channel is $\Delta T \approx 2 \mathrm{~K}$ above the lattice temperature $T=1.65 \mathrm{~K}$. This illustrates that the thermopower of a QPC is quite sizeable. In fact, one may use a QPC to measure locally the electron temperature, as we will now show.

\section{Peltier coefficient of a quantum point contact}

A measurement of the Peltier coefficient and the thermal conductance of a QPC requires a means of detecting the heat flow $Q$ through the constriction. We recently employed [5] the QPC thermometer technique for this purpose. Basically, the devices consist of a similar channel as used in the thermopower, but now fitted with a central QPC, defied by gates $B$ and E [Fig. 2(a)]. As a result of the heat flow, the electron temperature close to QPC BE will change by an amount $\delta T$. The temperature changes are detected by measuring the thermovoltages $V_{5}-V_{1}$ and $V_{4}-V_{2}$ across additional QPC's (defined by gates $\mathrm{A}$ and $\mathrm{B}$, and $\mathrm{B}$ and $\mathrm{C}$, respectively) situated at a distance $d$ from QPC BE. [The electrical conductance of the thermometer point contacts is adjusted in between at $G \approx 1.5 \times 2 e^{2} / h$ ), so that the thermopower $S \approx 40 \mu \mathrm{V} / \mathrm{K}$ has its maximum value $[4,9,10]$.] Note that the voltages $V_{5}-V_{1}$ and $V_{4}-V_{2}$ are positive if the electron-gas temperature in the channel exceeds that in the region behind the QPC's.

Figure 2(a) indicates the geometry used for a measurement of the Peltier coefficient of QPC BE. We use ohmic 


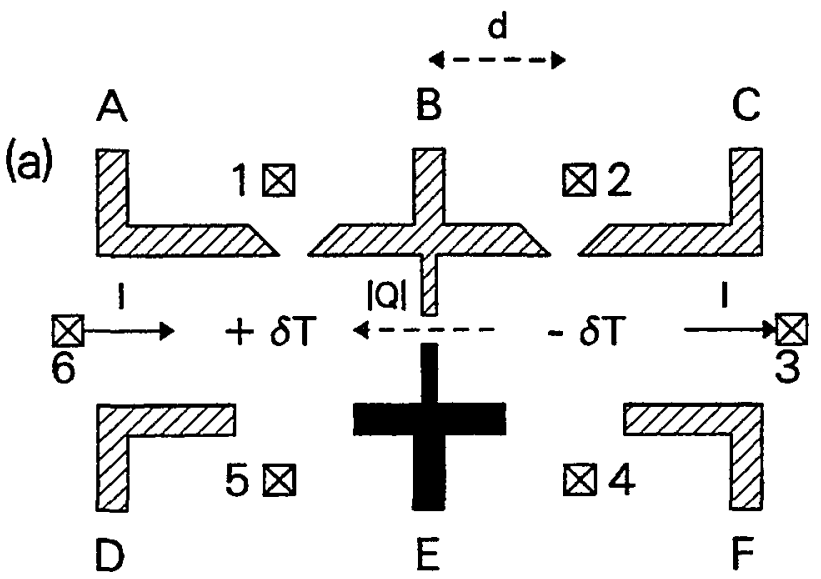

(b)

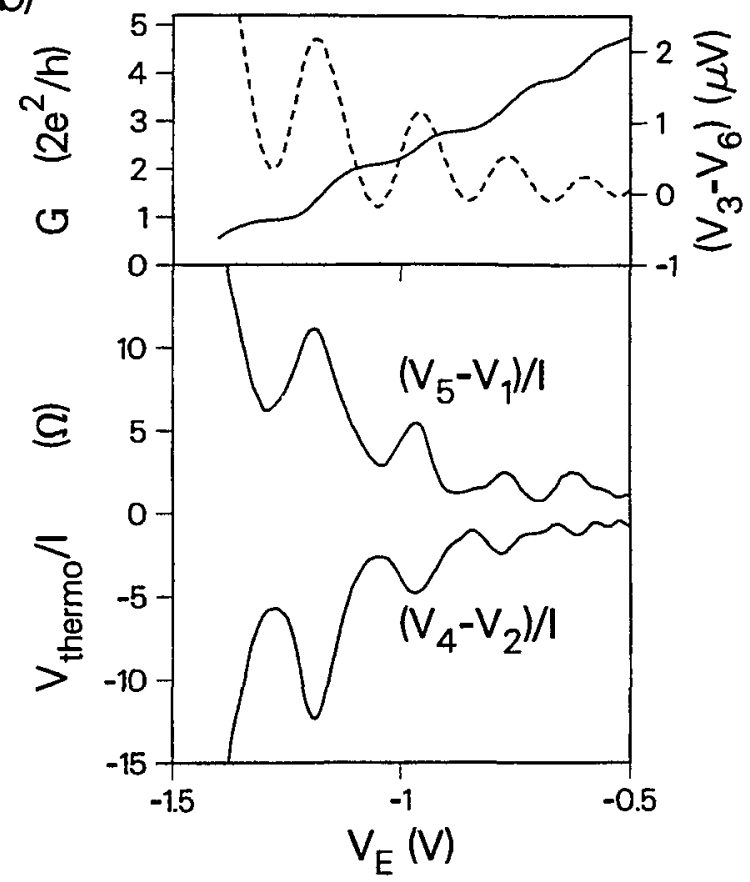

voltage $V_{E}$ in the bottom panel in Fig. 2(b). The experiment was made using a voltage bias; to compensate for the resulting $V_{\mathrm{E}}$-dependence of the current $I$ we have divided the measured thermovoltages by $I$. Oscillations in both $\left(V_{5}\right.$ $\left.-V_{1}\right) / I$ and $\left(V_{4}-V_{2}\right) / I$ are clearly visible. The oscillation maxima are aligned with the steps between conductance plateaux, shown for comparison in the top panel of Fig. 2(b) (solid line). The Peltier signals $\left(V_{5}-V_{1}\right) / I$ and $\left(V_{4}-V_{2}\right) / I$ are of opposite sign, consistent with heating occurring in the left, and cooling in the right section of the channel (for the current direction used). It is instructive to compare the Peltier signals with experimental data on the oscillatory thermovoltage $\left(V_{3}-V_{6}\right)$ of QPC BE (obtained by passing a $\mathrm{AC}$ current of $1 \mu \mathrm{A}$ from Ohmic contacts 2 to 4 ), shown as the dashed line in the top panel of Fig. 2(b). The presence of similar oscillations in the Peltier and thermovoltage signals is a direct consequence of the Kelvin-Onsager relation $\Pi=S T$ (eq. (3)).

\section{Thermal conductance of a quantum point contact}

The electrical circuit used for a measurement of the thermal conductance of QPC BE is shown in Fig. 3(a). We pass an AC current $I$ through the right section of the channel only
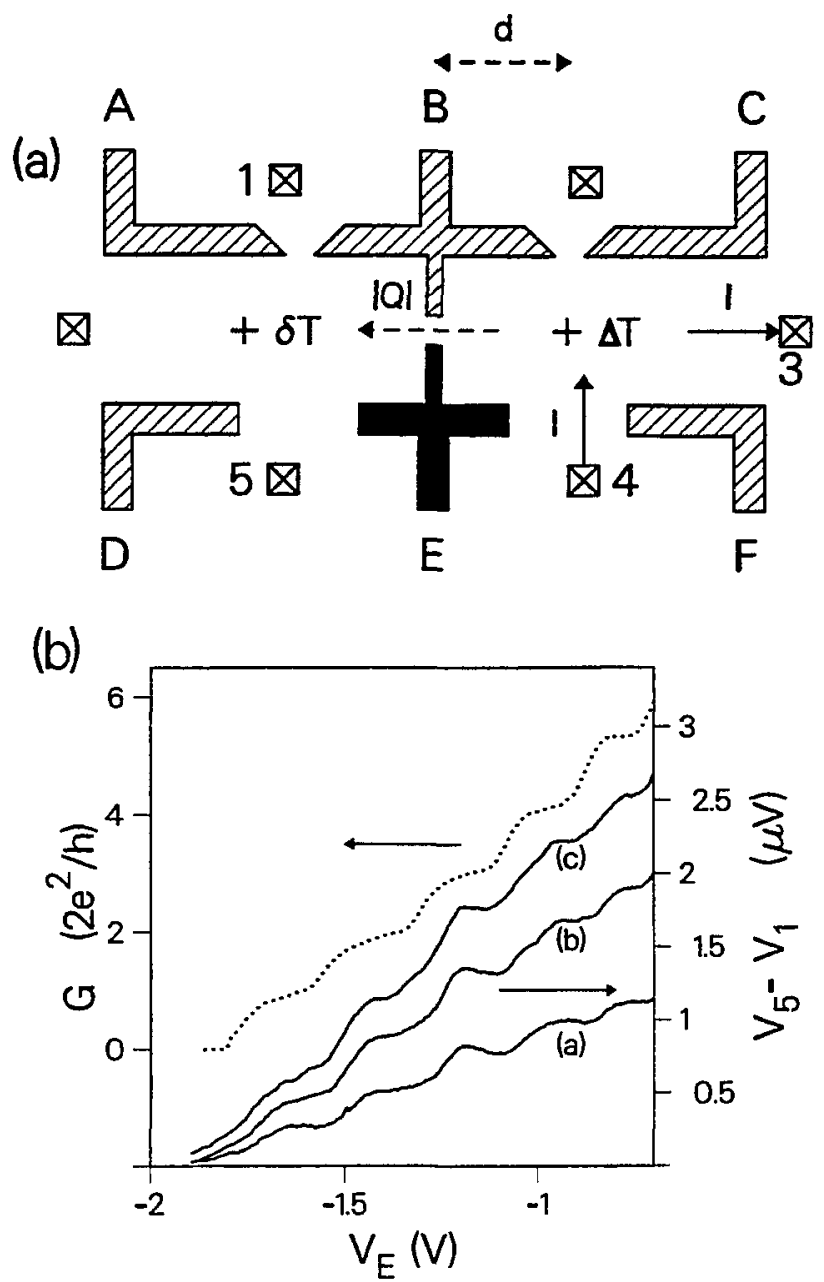

Fig. 2. (a) Sample and wire attachment to study the Peltier coeficient of central point contact $B E$. The total length of the channel is $40 \mu \mathrm{m}$; $d=5 \mu \mathrm{m}$. (b) The bottom panel shows the thermo-electric voltages measured to the left $\left(V_{5}-V_{1}\right)$ and right $\left(V_{4}-V_{2}\right)$ of QPC BE, divided by the current $(I \sim 0.2 \mu \mathrm{A})$, as a function of $V_{\mathrm{E}}$. For comparison, conductance quantization (solid line) and thermovoltage oscillations (dashed line) of QPC BE are plotted in the top panel. In the bottom panel, a positive voltage implies an increase in electron temperature in the channel.

contacts 6 and 3 as current source and drain, respectively, and scan the voltage on gate $E$ [black in Fig. 2(a)]. QPB BE is the main resistive element in the current path. The contact resistance of the QPC will lead to power dissipation on both sides of QPC BE. However, since for the current direction indicated, the right part of the channel is at a higher electrochemical potential than the left part, the dissipation is slightly larger in the left part of the channel. This imbalance corresponds to the Peltier heat flow $Q=\Pi I$ through QPC $B E$, on which we expect to observe a quantum size effect. The Peltier signal can be discriminated from the much larger dissipation background by using an $\mathrm{AC}$ current and a lock-in detector tuned to the fundamental frequency. (Note that the power dissipation gives a signal on the lock-in at twice the AC frequency.)

The results of such an experiment, obtained for a $d=5 \mu \mathrm{m}$ sample at $I \simeq 0.2 \mu \mathrm{A}$, are plotted versus gate

Fig. 3. (a) Sample and wire attachment to study the thermal conductance of central point contact BE. (b) Electrical conductance (dotted line) of QPC $\mathrm{BE}$ and thermovoltage $V_{5}-V_{1}$ as a function of the voltage on gate $\mathrm{E}$ (sample with $d=1.5 \mu \mathrm{m}$ ). An AC current $I$ is passed between ohmic contacts 3 and 4 . The thermovoltage is detected at the second harmonic frequency of $I$. Results are shown for $I=1.0 \mu \mathrm{A}$ [curve (a)], $1.3 \mu \mathrm{A}$ (b), and $1.5 \mu \mathrm{A}(\mathrm{c})$. 
(using Ohmic contacts 3 and 4). Through current heating, the electron gas temperature in this part of the channel is increased by $\Delta T$. The temperature difference $\Delta T$ across the central QPC (BE) gives rise to a heat flow $Q$ through this point contact. This causes a small temperature rise $\delta T \ll \Delta T$ in the electron gas in the left section of the channel, which we detect by measuring the thermovoltage $V_{5}-V_{1}$, as before. Figure 3(b) shows plots of the measured thermovoltage $V_{5}-V_{1}$ as a function of $V_{\mathrm{E}}$, for a $d=1.5 \mu \mathrm{m}$ sample, and constant heating currents of 1.0 [curve (a)], 1.3 (b), and $1.5 \mu \mathrm{A}(\mathrm{c})$. The thermovoltage scales with $I^{2}$, as expected. Sequences of plateaux in the thermovoltage are clearly visible, and each plateau lines up with a quantized conductance plateau of the point contact (dotted curve). This result demonstrates the occurrence of a quantum size effect in the thermal conductance $\kappa=-Q / \Delta T$ of a $Q P C$, similar to that in $G$. One would expect this similarity on the basis of the Wiedemann-Franz relation,

$\kappa \approx L_{0} T G$

where $L_{0} \equiv k_{\mathrm{B}}^{2} \pi^{2} / 3 e^{2}$ is the Lorenz number. Our experiment confirms that the Wiedemann-Franz relation holds approximately for a practical QPC. For an ideal QPC, one expects deviations, see [10]. We refer to Ref. [5] for a discussion of the magnitude of the experimental data on the Peltier coefficient and thermal conductance of a QPC.

This concludes our discussion of thermo-electric phenomena in the quantum-ballistic transport regime. As a final example of the current-heating technique, we now discuss a measurement of the thermopower of a quantum dot in the Coulomb-blockade, or single-electron tunneling regime.

\section{Thermopower of a quantum dot}

Single-electron tunneling is the dominant mechanism governing the transport properties of a quantum dot that is weakly coupled to reservoirs by tunnel barriers. At temperatures $T$ such that $k_{B} T \ll e^{2} / C$, with $C$ the capacitance of the dot, it leads to Coulomb-blockade oscillations in the conductance as a function of the voltage applied to a capacitively coupled gate electrode [2]. Whereas the conductance has been studied extensively, the thermoelectric properties of a quantum dot have received less attention. Recently, Beenakker and Staring developed a theory for the thermopower of a quantum dot in the Coulomb-blockade regime [12], which predicts sawtooth-like oscillations in the thermopower as a function of the Fermi energy in the reservoirs.

In order to observe these oscillations, we employ again [6] a sample [Fig. 4(a)] where current heating of the electrons in a narrow channel (defined by gates $B, C, A$, and D) provides the hot-electron reservoir. Gates $A, D$, and $F$ define two tunnel barriers with conductances of about $0.1 e^{2} / h$ each, leading to the formation of a quantum dot, whose potential can be varied by scanning the voltage on gate $V_{E}$. Analogous to the previous experiments, we measure the thermovoltage as a transverse voltage $V_{2}-V_{1}$, which equals to the difference in thermovoltages across the dot and reference QPC. In Fig. 4(b) we compare measurements of the Coulomb-blockade oscillations as a function of $V_{E}$ in the thermovoltage (solid) and conductance (dashed) of the dot, at a lattice temperature of $T=45 \mathrm{mK}$. Clearly, the (a)

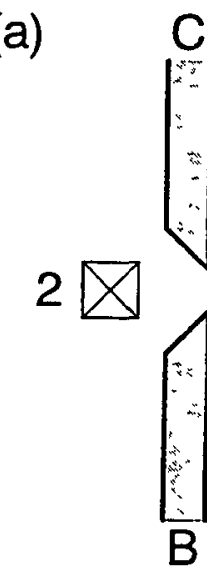

(b)

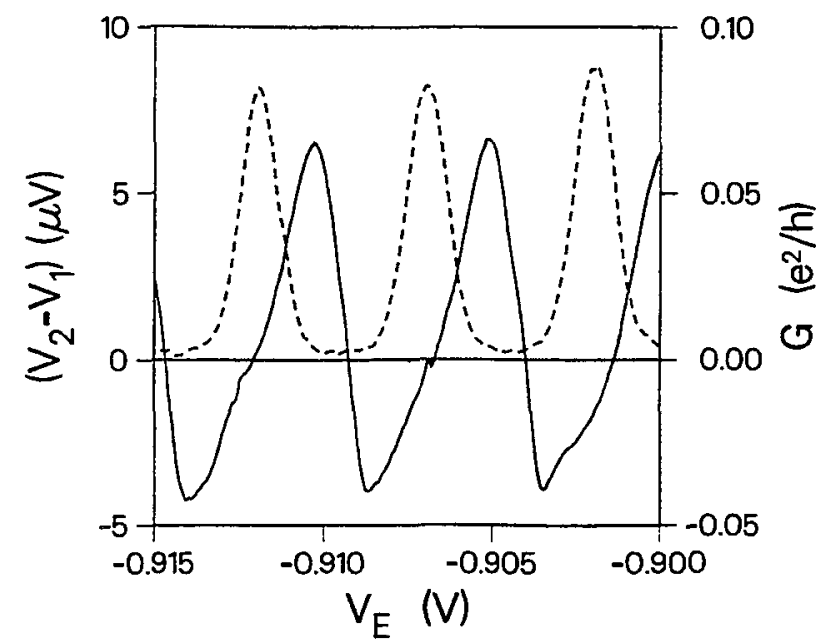

Fig. 4. (a) Schematic top-view of a $0.7 \times 0.8 \mu \mathrm{m}^{2}$ quantum dot adjacent to a $2 \mu \mathrm{m}$ wide, $20 \mu \mathrm{m}$ long channel. Gates, A, D, and F (hatched) define individually adjustable tunnel barriers, and gate $\mathrm{E}$ (black) controls the electrostatic potential of the dot; the gaps between gates $D$ and $E$, and between gates $E$ and $F$, are pinched off in the experiment. (b) Thermovoltage $V_{2}$ $-V_{1}$ at a heating current of $58 \mathrm{nA}$ (solid) and conductance (dashed) as a function of $V_{E}$ at a lattice temperature of $T=45 \mathrm{mK}$.

thermovoltage $V_{2}-V_{1}$ (and therefore the thermopower of the dot) oscillates periodically. The period is equal to that of the conductance oscillations, and corresponds to depopulation of the dot by a single electron. In contrast to the conductance oscillations, which consist of a series of symmetric peaks separated by gate-voltage regions where the conductance is suppressed, the thermovoltage oscillations have a distinct sawtooth lineshape. In addition, the conductance peaks are approximately centered on the positive slope of the thermovoltage oscillations, with the steeper negative slope occurring in between two conductance peaks. These data comprise a clear experimental demonstration of the key characteristics of the thermopower oscillations of a quantum dot. We refer to Ref. [6] for a full description of the experiments.

\section{Conclusions}

In conclusion, we have reviewed several experiments where current heating is utilized to create hot electron reservoirs in semiconductor nanostructures. We have shown how the technique can be employed to demonstrate quantum size 
effects in the thermoelectric transport coefficients of a 'quantum point contact, and to study the sawtooth-shaped thermopower oscillations of a quantum dot in the Coulomb-blockade regime.

\section{Acknowledgements}

This work was done in collaboration with B. W. Alphenaar, M. J. P. Brugmans, R. Eppenga and Th. Gravier. O. J. A. Buyk and M. A. A. Mabesoone provided expert technical assistance. The heterostructures were grown by C. T. Foxon at the Phillips Research Laboratories in Redhill (Surrey, UK).

\section{References}

1. Beenakker, C. W. J. and van Houten, H., Solid State Phys. 44, 1 (1991).

2. van Houten, H., Beenakker, C. W. J. and Staring, A. A. M., in: "Single Charge Tunneling" (Edited by $H$. Grabert and M. H. Devoret) (NATO ASI Series B, Vol. 294) (Plenum, New York 1992).

3. Butcher, P. N., J. Phys. Condens. Matter 2, 4869 (1990).
4. Molenkamp, L. W., van Houten, H., Beenakker, C. W. J., Eppenga, R. and Foxon, C. T., Phys. Rev. Lett. 65, 1052 (1990).

5. Molenkamp, L. W., Gravier, Th., van Houten, H., Buyk, O. J. A., Mabesoone, M. A. A. and Foxon, C. T., Phys. Rev. Lett. 68, 3765 (1992).

6. Staring, A. A. M., Molenkamp, L. W., Alphenaar, B. W., van Houten, H., Buyk, O. J. A., Mabesoone, M. A. A., Beenakker, C. W. J. and Foxon, C. T., Europhysics Lett., Europhysics Lett. 22, 57 (1993).

7. van Wees, B. J., van Houten, H., Beenakker, C. W. J., Williamson, J. G., Kouwenhoven, L. P., van der Marel, D. and Foxon, C. T., Phys. Rev. Lett. 60, 848 (1988); Wharam, D. A., Thornton, T. J., Newbury, R., Pepper, M., Ahmed, H., Frost, J. E. F., Hasko, D. G., Peacock, D. C., Ritchie, D. A. and Jones, G. A. C., J. Phys. C21, L209 (1988).

8. Sivan, U. and Imry, Y., Phys. Rev. B33, 551 (1986).

9. Streda, P., J. Phys. Condens. Matter 1, 1025 (1989).

10. van Houten, H., Molenkamp, L. W., Beenakker, C. W. J. and Foxon, C. T., Semicond. Science and Technol. 7, B215 (1992).

11. Proetto, C., Phys. Rev. B44, 9096 (1991); Solid State Commun. 80, 909 (1991).

12. Beenakker, C. W. J. and Staring, A. A. M., Phys. Rev. B46, 9667 (1992). 